\title{
Correction to: The Italian multiple sclerosis register
}

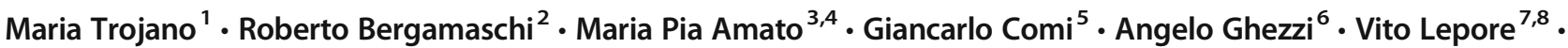 \\ Maria Giovanna Marrosu ${ }^{9}$. Paola Mosconi ${ }^{8}$. Francesco Patti ${ }^{10}$. Michela Ponzio ${ }^{11}$. Paola Zaratin ${ }^{11}$. \\ Mario Alberto Battaglia ${ }^{11,12} \cdot$ Italian Multiple Sclerosis Register Centers Group
}

Published online: 28 February 2019

(C) Fondazione Società Italiana di Neurologia 2019

\section{Correction to: Neurological Sciences (2019) 40:155-165 https://doi.org/10.1007/s10072-018-3610-0}

Unfortunately in the original publication, the affiliation of the author Maria Pia Amato was incorrect. The author inadvertently missed out to include her second affiliation.

The correct affiliation for Maria Pia Amato should read as follows:

${ }^{3}$ Department NEUROFARBA, MS Center AOU Careggi, University of Florence, Florence, Italy

${ }^{4}$ IRCCS Fondazione Don Carlo Gnocchi

Publisher's note Springer Nature remains neutral with regard to jurisdictional claims in published maps and institutional affiliations.

The online version of the original article can be found at https://doi.org/ $10.1007 / \mathrm{s} 10072-018-3610-0$

\section{Maria Trojano}

maria.trojano@uniba.it

$\triangle$ Mario Alberto Battaglia registroitalianosm@aism.it

1 Department of Basic Medical Sciences, Neuroscience and Sense Organs, University of Bari, "Aldo Moro" Policlinico Italy, Piazza Umberto I, Bari, Bari, Italy

2 Multiple Sclerosis Center, IRCCS Mondino Foundation, Pavia, Italy

3 Department NEUROFARBA, MS Center AOU Careggi, University of Florence, Florence, Italy

4 IRCCS Fondazione Don Carlo Gnocchi, Milan, Italy

5 Neurology Department and INSPE-Institute of Experimental Neurology, Vita-Salute San Raffaele University, Milan, Italy
6 Centro Studi Sclerosi Multipla, Ospedale di Gallarate, Gallarate, Va, Italy

7 Coreserach Center for Outcomes Research and Clinical Epidemiology, Pescara, Italy

Istituto di Ricerche Farmacologiche Mario Negri IRCCS, Milan, Italy

9 University of Cagliari Centro Sclerosi Multipla, Cagliari, Italy

10 Department of Neurosciences G.F. Ingrassia, University of Catania, Catania, Italy

11 Italian Multiple Sclerosis Foundation, Via Operai 40, Genoa, Italy

12 Department of Life Sciences, University of Siena, Siena, Italy 